\title{
Multiple Pregnancies Are Not Often
}

\section{Siniša Franjić*}

Independent Researcher, Croatia

Correspondence to: Siniša Franjić, Independent Researcher, Croatia; E-mail: sinisa.franjic@gmail.com Received date: July 1, 2020; Accepted date: July 12, 2020; Published date: July 19, 2020

Citation: Siniša Franjić (2020) Multiple Pregnancies Are Not Often. J Obst Gynecol Surg. 1(1): pp. 1-4.

Copyright: @2020 Siniša Franjić. This is an open-access article distributed under the terms of the Creative Commons Attribution License, which permits unrestricted use, distribution and reproduction in any medium, provided the original author and source are credited.

\section{ABSTRACT}

When more than one fetus develops in the uterus of a woman, such a pregnancy is called multiple. Physiologically, the uterus of a woman is scheduled to give birth to one fetus at a time, so multiple pregnancies are considered rare. Multiple pregnancies are not so common in the world, but with more frequent fertilization by assisted methods and the treatment of female infertility, there is a greater number of ovulations than once, so multiple pregnancies are possible. However, it should be noted that multiple pregnancies can be associated with a high risk of premature pregnancy, early delivery and miscarriage, and even intrauterine death of one of the fetuses.

\section{Keywords:}

Multiple Pregnancy, Twin Pregnancy, Reduction, Ultrasound.

\section{Introduction}

The birth of the second twin has always been recognized as a hazardous procedure leading to perinatal loss and morbidity [1]. Complications of the delivery process of the second twin have led to several legal actions. The claims are usually based on the failure to diagnose the presence of a twin pregnancy or the difficulties in the delivery of the second twin.

For example, if the second twin, after rupturing of the membranes, presents as a transverse lie, then delivery can only be affected by the internal podalic version and breech extraction of the child. In practice, as the cervix is fully dilated, and as the presenting part should be easily accessible, delivery can be rapidly effected. However, if the child shows evidence of trauma or asphyxia, a claim is commonly based on delay in taking action to effect delivery, or allegations are made that the child should have been delivered by Caesarean section. As a result, it is becoming increasingly common to see the second twin delivered by Caesarean section, a procedure that has not proven to be of benefit and carries distinct risks to the mother. It would be safer to routinely deliver all twins by elective Caesarean section.

\section{Pelvic structure}

Reduction in family size has for centuries occurred as societies urbanize and develop economically [2]. While it is necessary to have many children in an agrarian economy to help with farming, caring for livestock, and caring for younger children and elders while parents work to provide food in societies that produce industrial goods or post-industrial services, the need for large families dissipates. Also, as societies develop and health care improves, more children live past infancy and childhood, so it is not as necessary to bear many children for enough to survive to adulthood to provide for parents as they age.

To this day, in less-developed countries in Asia, Africa, and the Americas, it is not uncommon for women to endure more than a dozen pregnancies, resulting in between 6 and 10 live births, but only have two to four children live beyond the age of five or six. Thousands of children die every week in these countries of bacterial, viral, and parasitic infections that no longer exist in the industrialized world and could easily be treated if the medicines were available. Multiple pregnancies and births put a huge burden on the health of women in these societies, many of whom have their first pregnancy while still in their early or mid-teens.

Many women die during childbirth, and many babies are stillborn because proper nutrition and prenatal health care are lacking. Other women are permanently crippled by rectovaginal fistulas, which occur when the skin that separates the rectum and the vagina becomes torn during childbirth. This is a frequent problem for younger teens, whose pelvic structure is not always developed enough for a baby to exit the uterus through the vagina, and who often give birth in rural health clinics or at home without proper obstetric care. If these fistulas are not corrected and they are rarely corrected in less-developed countries these girls and women may become incontinent (unable to control their flow of urine or feces). The constant dribble of urine and feces can make them susceptible to disease, and the odor can make them outcasts in their communities.

Under normal conditions, the uterus enlarges uniformly and its height is proportionate to the period of pregnancy [3]. The anteverted fundus of the pregnant uterus becomes just palpable at the symphysis at about 10 weeks. By the 12th week, it is palpable above the symphysis; it is halfway between the symphysis and umbilicus at 16 weeks and the level of the umbilicus at 20 weeks. The fundus then rises one 4th of the way to the xiphisternum each month until the ninth, when at term it sinks to the level it occupied in the 8th month but there is a falling forward of the fundus. Determining the duration of pregnancy by this method cannot be accurate because of the inconsistency of the location of the umbilicus, thickness of the abdominal wall, the amount of liquor amnii, the size of the foetus and the possibility of multiple pregnancies. Despite these, at times the estimate is fairly accurate.

The height of the uterus is usually smaller when compared to the period of amenorrhoea in cases of ectopic gestation, retroverted gravid uterus, intrauterine death of the foetus and oligohydramnios. On the other hand, it is larger in a vesicular mole, hydramnios, multiple pregnancies, and concealed accidental hemorrhage and tumors complicating pregnancy.

Antenatal 
In natural conceptions 1:80 results in twin pregnancy. 1:6400 conceptions results in triplets, and 1:512 000 results in quadruplets [4].

Where an average incidence of triplets in a maternity unit delivering 3000 babies a year might result in a triplet birth every 2 years, it is becoming more common to see two to three such births in a year.

- Early diagnosis so relevant information and support can be organized

- Determination of whether the twins are identical or not (zygosity)

- Chorionicity: if the pregnancy has only one chorion there is a three- to fivefold higher risk of perinatal mortality. This can be determined from a scan in the first trimester

- Multiple pregnancies can be shorter than a single pregnancy. The average is 37 weeks for twins, 34 weeks for triplets, and 32 weeks for quads

- Identical or monochorionic twin pregnancies should have a scan every 2 weeks from diagnosis to look for discordant growth patterns; a sign of feto-fetal transfusion syndrome

- Non-identical twin pregnancies should have a scan every 4 weeks to monitor growth

- Regular measurement of maternal haemoglobin levels and iron supplements if needed

- The mother needs additional support both to prepare her for the birth and also the care and feeding of the babies

- The mother will need to pay particular attention to her dietary needs, the need for rest, and the possibility that she may have to stop work earlier than intended

\section{Twin pregnancy}

Seventy percent of twins are fraternal, resulting from fertilization of two separate ova by two different sperm [5]. Fraternal twins are no more alike than any other brothers and sisters, but they share a family resemblance because they are born of the same parents. Each fertilized ovum implants separately, and each twin forms its placenta and fetal membranes. Frequently, the margins of the two placentas grow together and fuse, but each fetus remains enclosed within its amnion and chorion. A fused placenta of this type is called a diamnionic dichorionic placenta. Thirty percent of twins are identical and result from the splitting of a single fertilized ovum. Splitting may occur at various times after fertilization. In about 30 percent of monozygotic twin pregnancies, the fertilized ovum splits before the inner cell mass forms, which is on the third day after fertilization. Each half of the zygote implants separately forms a complete embryo and develops its placenta. The two placentas may remain separate or may become fused to form a diamnionic dichorionic placenta in the same manner as that for fraternal twins.

More commonly (in almost 70 percent of monozygotic twin pregnancies), the inner cell mass divides after the blastocyst has formed but before implantation takes place. In this instance, each half of the inner cell mass forms a complete embryo and develops its amnion and yolk sac, but both develop within a single chorionic cavity. This gives rise to a diamnionic monochorionic placenta.

Rarely, the inner cell mass divides after the amnionic sac has already formed. When this occurs, the two embryos develop within a single amnionic cavity and form a monoamnionic monochorionic placenta. If the division of the inner cell mass is incomplete, conjoined twins (Siamese twins) are formed.

In the past, it was thought that intrauterine growth in twin pregnancies began to fall below that of singletons at approximately the 30th week [6]. However, more recent observations indicate that ACs (abdominal circumference) of the twin falls below those of singleton as early as 15 weeks' gestation. Of importance is the observation that, as pregnancy advances, the differences in ACs between twin and singleton fetuses widen from approximately $1.0 \mathrm{~cm}$ to $2.0 \mathrm{~cm}$ at 31 and 38 weeks, respectively. Likewise, the differences between the actual birth weights of singleton and twin fetuses increase with advancing gestation, from approximately $150 \mathrm{~g}$ to $610 \mathrm{~g}$ at 31 and 40 weeks, respectively. Of particular interest is that the 50th weight centile of twin fetuses crosses the 10th weight centile of singleton fetuses at approximately the 37th pregnancy week, and subsequently falls below the 10th centile of singletons.

\section{Multiple pregnancy}

Multiples grow in utero to the same extent as singletons until about 28 weeks, thereafter, growth curves show a clear decelerating trend compared with that of singletons [7]. The higher risk of delivering Low Birth Weight (LBW) infants in multiple births is well known, as is the advantage for the multiparous patient. Analysis of population-based data found that overall, the risk of having at least one Very Low Birth Weight (VLBW, <1500g) infant was 1:5 among nulliparous women and 1:12 among multiparous women. The risk of having two VLBW twins among nulliparas (1:11) was double that of multiparas (1:22). A similar trend and similar frequencies, but for extremely LBW $(<1000 \mathrm{~g})$ babies, were found in the analysis of triplets.

The most common growth aberration in multiples is birth weight discordance (relative growth restriction). Birth weight discordance occurs whenever a significant difference exists in birth weights between the larger and the smaller fetus/infant of a multiple pregnancy set. One rarely finds that all members of the set have the same birth weight as some variation is expected between siblings and therefore the magnitude of the difference the degree of discordance must be incorporated in the definition. At present, the percent definition is usually employed, whereby the birth weight disparity is calculated as a percentage of the larger infant. Because the definition does not refer to the actual size of the twins, the same degree of discordance (eg. 20\%) may be assigned to a twin pair weighing 1500 and $1200 \mathrm{~g}$ and to a pair weighing 3000 and $2400 \mathrm{~g}$. The analysis shows that about $75 \%$ of twins exhibit less than $15 \%$ discordance, about $20 \%$ are $15-25 \%$ discordant, and about $5 \%$ are more than $25 \%$ discordant.

The definition of birthweight discordance is even more complex in triplets. Using the same percent definition as used for twins will ignore the middle-sized triplet. Therefore, the true estimation of the inner triplet relationship requires a different approach, in which the middle-sized triplet is defined with the difference between the larger and the smaller triplets.

\section{Reduction}

The increased use of fertility treatment has led to higher numbers of multiple pregnancies [8]. Careful monitoring of ovulation induction and a reduction in the maximum number of embryos replaced in in vitro fertilization treatment can help 
to reduce the number of multiple pregnancies, but despite concerted efforts to reduce the number, this cannot be avoided in all cases. High order multiple pregnancies are known to be associated with higher rates of mortality and morbidity for mothers and their babies. The greater the number of fetuses the greater the likelihood of preterm delivery and adverse consequences for the health and development of each baby. The risk may be reduced by 'selective reduction', or multifetal pregnancy reduction, which involves killing one or more of the fetuses in utero to give the others a greater chance of a healthy outcome. There is evidence that the perinatal and obstetric outcomes after reduction of four or more fetuses are improved but whether the same applies to triplets reduced to twins remains controversial. Some also see the procedure as posing medical, ethical, and psychosocial problems, not least because of the paucity of information about how women and their partners cope with the experience and its aftereffects. The limited research that has been published suggests that most mothers considered that they had made the right decision regarding multi-fetal pregnancy reduction but there are undoubtedly complex psychological factors in the short and long term.

\section{Ultrasound}

Unlike the imaging techniques that depend on the detection of electromagnetic radiation as part of the imaging process, ultrasound is a technique for mapping the reflected echoes produced by high-frequency sound waves transmitted into the body [5]. Echoes are reflected wherever there is a change in the density of the tissue. The reflected waves are recorded on a detector, and visual images are produced, generally on a small monitor. Unlike the complex equipment needed for CT (Computer Tomography) or PET (Positron Emission Tomography) scans, ultrasound devices commonly use a single handheld transducer to produce the ultrasound and record the reflected echoes. The transducers may be moved over any external area of the body (using a water-soluble gel to couple the ultrasound to the area of study) to produce a real-time image of internal organs under and within range of the acoustical beam as the transducer is moved. Although there are limits to the depth of penetration of ultrasound from the body surface (and hence to what internal areas of the body can be imaged), ultrasound transducers are small enough to be introduced into the rectum (to image the prostate in males), the vagina (for gynecological investigations in females), and the esophagus (to image abdominal organs such as the pancreas). This method is widely used to study the uterus during pregnancy because it does not require the use of potentially harmful radiation and poses no risk to the fetus.

The technique can be used to determine the position of the placenta and the fetus within the uterus; it also can identify some fetal abnormalities and detect twin pregnancies.

Two main types of ultrasound are in common use: continuous ultrasound is used to detect moving structures such as the fetal heart, whereas pulse ultrasound is used to outline structures within the uterus [9]. In obstetrics, ultrasound is used for the screening and diagnosis of fetal age, multiple pregnancies, intrauterine growth retardation, and fetal malformations including craniospinal defects such as anencephaly, cardiac defects, and musculoskeletal, gastrointestinal, and renal abnormalities. Diagnostic ultrasound has no known risks to the fetus or mother and produces little anxiety in low-risk women. Women tend to view scans as benign procedures allowing them to confirm the baby is healthy. Scans may promote maternalfetal bonding and may lead to a decrease in behaviors such as smoking and drinking, especially in women who receive a high level of feedback during the ultrasound examination.

\section{Cesarean section}

The incidence of twins, triplets, and high-order multifetal pregnancies has increased significantly since the introduction of artificial stimulation of ovulation and in vitro fertilization [10]. These pregnancies are at a higher risk than singletons for perinatal morbidity and mortality. Although much has been written about the mode of delivery for these gestations, there is little consensus over whether the cesarean section improves the outcome.

Twin gestations make up approximately $1 \%$ of all pregnancies. Although dizygotic twinning varies with race, age, parity, and, other factors, the monozygotic twinning rate is constant, occurring at a rate between 3 and 4 per 1000 births. Morbidity and mortality are increased remote from delivery. Complications include spontaneous abortion, intrauterine growth retardation, preeclampsia, placental abnormalities, and preterm labor.

There is consensus regarding the safety of vaginal delivery of twins when both twins are vertex, regardless of gestational age. Cesarean section should be reserved for usual indications, that is, fetal stress or distress and cephalopelvic disproportion.

Cesarean section has been recommended by some investigators as the mode of delivery in vertex/nonvertex presentations to avoid hazardous breech extraction of the second twin. During the past several years numerous studies have suggested, however, that the second, nonvertex twin weighing more than $1500 \mathrm{~g}$ can be delivered vaginally with as much safety as by cesarean section.

\section{After birth}

Twin/triplet births are considered high risk [11]. These infants are at risk of being premature or smaller relative to their gestational age. An ultrasound taken during pregnancy may be useful in determining the major issues in the infant's development. Multiple births have climbed significantly in the last 30 years, achieving unprecedented numbers in twins, triplets, and other higher-order multiples. Contributing to these rising rates has been the trend to delay childbearing and the increased interest in infertility therapy and assisted reproductive technologies.

\section{Discussion}

In multiple pregnancies, it is important to ensure that each of the babies gets adequate care after birth. Anyone of the infants may have problems that need immediate intervention. About $2 \%$ of twins are born with major structural deformities, a condition that is higher in prevalence in same-sex twins. The most frequently encountered abnormalities are cardiac malformations, neural tube defects, facial clefts, and gastrointestinal anomalies. The rate of cardiac defects and gastrointestinal anomalies in multiples is twice the rate for singletons. The striking incidence of congenital anomalies in all twins is almost exclusively related to the higher rate of anomalies in monozygotic twins.

Some abnormalities are seen only in multiple gestations. 
Conjoined twins occur at a rate of $1 / 50,000$ to $1 / 100,000$ births, being three times more common in female fetuses than in male fetuses. The survival of conjoined twins is seen to be generally dependent on the extent of shared organs. If twin infants have twin-to-twin transfusion syndrome (or feto-fetal transfusion syndrome), the donor twin of the transfusion suffers retarded growth, anemia, pallidity, hypovolemia, and malnutrition. For this reason, these babies may have to be transferred to the intensive care unit. Parents' first encounter with the newborn is an important step in their relationship. Parents need guidance in this process.

\section{Conclusion}

Pregnancy with multiple fetuses and the birth of twins is not a common occurrence since the uterus of a woman is tuned to develop only one fetus. The age of the mother and the number of previous births have an impact on the incidence of multiple pregnancies, although hereditary factors are most important, especially when both parents are also twins. Certainly, inheritance plays a very significant role in terms of multiple pregnancies. Namely, there are parts of the world where the number of twin pregnancies is huge, and this cannot be explained by anything other than genetic predisposition. In marriages with twin pregnancies, in anamnesis of one or both spouses can be found ancestors or relatives who also had twins.

Conflict of Interest: There are no conflicts of interest.

\section{References}

1. Symonds EM (1991) Obstetrics and Gynaecology“ in Jackson, JP. (ed): A Practical Guide to Medicine and the Law, SpringerVerlag London Limited, London, UK, pp. 165.

2. Zonderman J. Shader L, Triggle DJ (2006) Birth Control Pills, Chelsea House Publishers, Infobase Publishing, New York, USA. pp. 31-33.
3. Vij K (2011) Textbook of Forensic Medicine and ToxicologyPrinciples and Practice, Fifth Edition, Elsevier, Reed Elsevier India Private Limited, New Delhi, India. pp. 328.

4. Medforth J, Batersby S, Evans M, et al. (2011) Oxford Handbook of Midwifery, Second Edition, Oxford University Press, New York, USA. pp. 170-171.

5. Reisner EG, Reisner HM (2017) Crowley's An Introduction To Human Disease-Pathology and Pathophysiology Correlations, Tenth Edition, Jones \& Bartlett Learning, Burlington, USA. pp. 17-483.

6. Blickstein I, Keith LG (eds) (2007) Prenatal Assessment of Multiple Pregnancy, CRC Press, Taylor \& Francis Group, Boca Raton, USA. pp. 111.

7. Blickstein I, Barak O (2019) Multiple pregnancies and births in Norwitz E, Zelop CM, Miller DA, et al. (eds) Evidence-Based Obstetrics and Gynecology, John Wiley \& Sons Ltd, Hoboken, USA. pp. 472.

8. Graves CR, Boehm F.H (1995) Twin Gestation and Multiple Births in Flamm BL, Quilligan EJ (eds) Cesarean SectionGuidelines for Appropriate Utilization, Springer-Verlag New York, Inc., New York, USA. pp. 125.

9. Robinson GE, Wisner KI (2001) Fetal Anomaly in Stotland NL, Stewart DE (eds) Psychological Aspects of Women's Health Care, The Interface Between Psychiatry and Obstetrics and Gynecology, Second Edition, American Psychiatric Press, Inc., Washington, USA. pp. 38.

10. Brannan S, Chrispin E, Davies M, et al. Medical Ethics TodayThe BMA's Handbook of Ethics and Law, Third Edition, WileyBlackwell, John Wiley \& Sons, Ltd, Chichester, UK. pp. 286.

11. Kuguoglu S, Yildiz H, Tanir MK, et al. (2012) Breastfeeding After a Cesarean Delivery" in Salim R (ed) Cesarean Delivery InTech, Rijeka, Croatia. pp. 139. 